\title{
Hallazgos endoscópicos y patológicos en pacientes con VIH y síntomas digestivos de un hospital universitario
}

\author{
Endoscopic and Pathological Findings in Patients with HIV and Digestive \\ Symptoms at a University Hospital
}

\author{
Luis Daniel Laguado V., MD, ${ }^{1}$ Reynaldo Mauricio Rodríguez Amaya, MD, ${ }^{2}$ Jaime Gómez C., MD, ${ }^{3}$ Rómulo Arturo Bonilla G., MD, ${ }^{4}$ \\ Gonzalo Rojas H., MD 4
}

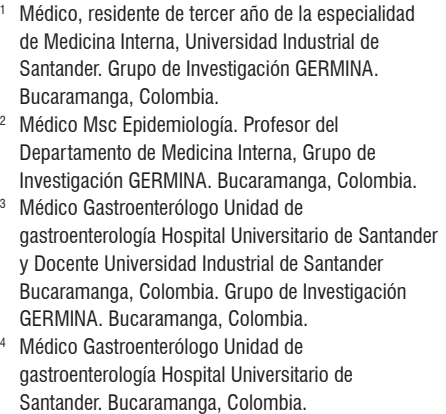

\begin{abstract}
Resumen
Introducción: el tracto gastrointestinal es un sitio frecuentemente afectado por el VIH; sin embargo, en la práctica clínica algunos hallazgos normales en la endoscopia pueden no serlo, lo cual conlleva a estos pacientes a no recibir tratamiento oportuno para patologías gastrointestinales. Métodos: estudio observacional descriptivo en pacientes con VIH que consultaron por síntomas digestivos y que requirieron endoscopia y/o colonoscopia durante el año 2014 en el Hospital Universitario de Santander. Se tomaron datos sociodemográficos, clínicos y paraclínicos. Se realizaron 41 endoscopias altas y 29 colonoscopias en 54 pacientes. A todo estudio se le practicó biopsia. Resultados: la edad promedio fue de 39 años, la sintomatología digestiva con mayor reporte fue la diarrea y las lesiones orales; $87 \%$ tenían conteo menor a 200 CD4, solo $24 \%$ reciben actualmente TARAE. Los diagnósticos más frecuentes por anatomía patológica diferentes a normalidad fueron: Candidiasis $(17 \%)$ en esófago, gastritis crónica moderada (26,7\%) en estómago y colitis crónica inespecífica moderada tanto en colon izquierdo (44,8\%) como en colon derecho $(51,7 \%)$. Conclusiones: los hallazgos endoscópicos y patológicos concuerdan con la frecuencia de presentaciones reportadas en la literatura, aunque no se encontraron neoplasias ni agentes infecciosos como micobacterias. Los oportunistas más frecuentes fueron la cándida y el citomegalovirus. Al confrontar el diagnóstico de normalidad entre el endoscopista y la anatomía patológica, solamente en esófago había una concordancia aceptable, a diferencia del estómago y el colon, donde la disparidad es evidente. Por lo anterior, en pacientes con $\mathrm{VIH} /$ SIDA que requieran endoscopia sería importante siempre considerar la toma biopsias de manera protocolizada.
\end{abstract}

Palabras clave

Sistema digestivo, endoscopia, infecciones por VIH, enteropatía por HIV.

\section{Abstract}

Introduction: Although the gastrointestinal tract is frequently affected by HIV, in clinical practice abnormalities related to HIV often appear to be normal when seen through an endoscope. The consequence is that these patients do not receive timely treatment for gastrointestinal diseases. Methods: This is an observational study of HIV patients who came to the hospital of the Universidad Industrial de Santander because of digestive symptoms that required either endoscopy or colonoscopy, or both ,during 2014. Socio-demographic, clinical and laboratory data were collected. A total of 41 upper endoscopies and 29 colonoscopies were performed in 54 patients. Biopsies were taken and analyzed in all cases. Results: The mean patient age was 39 years old, the most frequent digestive symptoms were diarrhea and oral lesions, $87 \%$ of these patients had CD4 counts below 200 , and only $24 \%$ currently receive HAART. The most frequent diagnoses were: esophageal candidiasis (17\%), moderate chronic gastritis in the stomach $(26.7 \%)$, and moderate chronic nonspecific colitis in the left colon $(44.8 \%)$ and in the right colon (51.7\%). Conclusions: Endoscopic and pathological findings are consistent with the frequencies of presentations of gastrointestinal pathologies reported in the literature except that no tumors or infectious agents such as mycobacteria were found. The most common opportunistic infections were Candida and Cytomegalovirus. Diagnostic agreement between the endoscopist and pathologist varied. There was had only fair agreement for the esophagus, but there were large disparities for the stomach and colon. Consequently, when HIVIAIDS patients require endoscopy, it is important that the protocol call for biopsy samples and analysis.

\section{Keywords}

Digestive System, endoscopy, HIV infections, HIV enteropathy. 


\section{INTRODUCCIÓN}

Según el Programa Conjunto de las Naciones Unidas sobre el VIH/SIDA (ONUSIDA) en el mundo 35 millones de personas conviven con el virus de la inmunodeficiencia humana (VIH). Dadas sus consecuencias e implicaciones se considera como un verdadero problema en salud pública. A su vez, se calcula que el $0,8 \%$ de los adultos de edades comprendidas entre los 15 y 49 años son seropositivos (1). En Colombia, para el año 2013, 45543 personas tenían VIH, lo que establece una prevalencia en la población general de $0,11 \%$ y en la población de 15 a 49 años de 0,16\% (2). Así mismo, las manifestaciones clínicas de la enfermedad por VIH afectan múltiples órganos y sistemas, estas alteraciones varían en severidad y pueden deberse a la replicación del VIH en el tejido infectado, infecciones oportunistas o efectos adversos de los medicamentos (3). El tracto gastrointestinal en particular es un sitio muy frecuentando por la infección por VIH, se reportan cifras hasta del $88 \%$ en pacientes que tenían al menos una anormalidad en la función gastrointestinal (4). También se ha descrito que la causa más frecuente de suspensión de la terapia antiretroviral altamente efectiva (TARAE) son las manifestaciones gastrointestinales; a su vez se ha recalcado el importante papel que desempeña la endoscopia en la era pos-TARAE (5).

Por otra parte, la endoscopia es una prueba diagnóstica invasiva que contribuye a esclarecer la etiología de la mayoría de las enfermedades gastrointestinales asociadas a la infección por VIH, sin embargo en algunas oportunidades es difícil encontrar un agente etiológico especifico, esto se explica parcialmente con la afinidad del virus por afectar los tejidos del sistema gastrointestinal, generando una pronunciada pérdida de linfocitos CD4 en la lámina propia de la mucosa intestinal $(6,7)$. Revisando la literatura disponible, son pocos los estudios en Latinoamérica y en el mundo en donde se ofrezca información sobre el reporte endoscópico y anatomo-patológico en pacientes con VIH. Por lo general los estudios al respecto ofrecen información sobre patologías muy específicas $(8,9)$. Por lo anterior, este estudio pretendió ilustrar los hallazgos clínicos endoscópicos y patológicos de una serie de pacientes con VIH que tienen como principal motivo de consulta los síntomas digestivos.

\section{MÉTODOS}

\section{Diseño y población del estudio}

El estudio fue realizado en la Unidad de Gastroenterología del Hospital Universitario de Santander en Bucaramanga,
Colombia. Se diseñó un estudio observacional, descriptivo, donde se recolectó una serie de pacientes que ingresaron al Hospital desde el 01 de enero hasta el 31 de diciembre de 2014. Dicho hospital es centro de referencia para la red pública en la región nororiental de Colombia. Como criterios de inclusión, los pacientes debían ser mayores de 18 años, tener diagnóstico de VIH confirmado con Western Blot y, al ingreso al Hospital, debían tener sintomatología digestiva como motivo de consulta principal. Igualmente, los pacientes debían tener indicación clínica por parte de la unidad de gastroenterología del hospital para la realización de endoscopia de vías digestivas altas o bajas. Entre los criterios de exclusión se consideraban aquellos pacientes que presentaran inestabilidad hemodinámica, alteraciones neurológicas o agitación psicomotora que impidan realizar los estudios endoscópicos o suministrar el consentimiento informado.

\section{Recolección de los datos}

Una vez el paciente cumplía con los criterios de inclusión, se procedió a diligenciar un cuestionario en donde se incluían variables sociodemográficas, clínicas y paraclínicas. Las variables clínicas abordaban información sobre los síntomas digestivos y también incluía información sobre el tratamiento previo recibido dada su condición de paciente con VIH. En cuanto a las variables paraclínicas se tuvieron en cuenta parámetros como hemograma completo $\mathrm{y}$ recuento de linfocitos CD4. A su vez, en el resultado de informe endoscópico se describían los hallazgos anormales y el diagnóstico endoscópico observado por el gastroenterólogo. Por último, se registró el diagnóstico patológico entregado por el servicio de patología de la Facultad de Salud de la Universidad Industrial de Santander (UIS).

\section{Realización del procedimiento endoscópico}

En cuanto a la preparación para realizar los procedimientos diagnósticos para colonoscopia, se aplicaron tres enemas orales y uno rectal (fosfato de sodio), dieta líquida el día anterior y suspender la vía oral el día del examen. Para endoscopia alta, el paciente debía tener ayuno desde la noche anterior ( 12 horas) y no haber recibido vía oral el día del examen. Los equipos utilizados para endoscopia y colonoscopia fueron: torre de endoscopia Olympus EVIS EXERA II CV-180/CVL-180; torre Olympus EVIS EXERA CV-145/CLV-160; endoscopios Olympus EVIS EXERA II GIF Type H180/CF Type H180AL y otro Olympus GIF Type Q150/EVIS CF Type Q145L. El procedimiento endoscópico se realizó en el servicio de 
gastroenterología por alguno de los tres gastroenterólogos del servicio, quienes realizaban el estudio endoscópico y el reporte del mismo de forma estandarizada.

En la endoscopia digestiva alta se exploró desde esófago hasta la segunda porción de duodeno, se reportaba el hallazgo de candidiasis esofágica con la escala de Kodsi y el hallazgo de esofagitis con la escala de Los Ángeles $(10,11)$; a los demás hallazgos anormales se les describían las características endoscópicas y se determinaban los diagnósticos endoscópicos. Una vez encontradas las lesiones, se tomaban las biopsias según criterio del endoscopista (mínimo cuatro), Si no habían lesiones macroscópicas y el estudio endoscópico era normal, por protocolo, se tomaban dos muestras de esófago distal y dos del estómago. En endoscopia digestiva baja se estudiaba desde el canal anal hasta el ciego, el hallazgo de colitis ulcerativa se informaba según la clasificación de Montreal (12), se determinaban los diagnósticos endoscópicos y se tomaban al menos cuatro biopsias de las lesiones. Ante el hallazgo macroscópico normal se tomaban por protocolo cuatro biopsias, dos en el colon derecho y dos en el colon izquierdo.

\section{Procesamiento y lectura de biopsias}

Las biopsias se fijaban en un frasco de boca ancha con formol bufferizado al $10 \%$, respetando la proporción mínima de 10:1 (formol/muestra), se rotulaban y se enviaban al laboratorio de patología con la indicación de realizar tinciónes de Hematoxilina eosina, Zieln Neelsen, Periodic Acid Schiff (PAS), Plata Metenamina y Giemsa, para lo cual se elaboró un sello para poder identificar en el servicio de patología las muestras de este estudio. Estas muestras fueron estudiadas según protocolo del departamento de patología de la UIS con tres observadores y realización de estudios complementarios según necesidad.

\section{Operacionalización de las variables}

En cuanto al recuento de células sanguíneas, al ingreso al hospital a todos los pacientes del estudio se les tomó un hemograma automatizado, la muestra fue analizada con un equipo SYSMEX XN-1000. De acuerdo a los valores generados por el hemograma, se definió como anemia la hemoglobina menor de $12 \mathrm{~g} / \mathrm{dL}$ en la mujer y de $13 \mathrm{~g} / \mathrm{dL}$ en el hombre, leucopenia como un conteo menor a 4000 leucocitos por $\mathrm{mm}^{3} \mathrm{y}$ trombocitopenia como un conteo de plaquetas menor a 150000 por $\mathrm{mm}^{3}$. Finalmente, en cuanto a la cuantificación de linfocitos CD4, durante la hospitalización a cada paciente se le realizó determinación del estado inmunológico mediante la cuantificación de linfoci- tos CD4 con el equipo de citometría de flujo CYTOMICS FC500 Beckman Counter.

\section{Análisis estadístico}

De acuerdo a su distribución, las variables cuantitativas fueron analizadas mediante promedios con su respectiva desviación estándar (DE) o medianas. Las variables cualitativas se presentan como proporciones con sus respectivos intervalos de confianza.

\section{CONSIDERACIONES ÉTICAS}

Este estudio cumple los lineamientos de la declaración de Helsinki y la legislación colombiana vigente (resolución 8430 de 1993) con respecto a las investigaciones en salud; además se obtuvo el consentimiento informado para la utilización de la información clínica y de exámenes de laboratorio de los pacientes incluidos en este estudio. De igual manera, el presente estudio fue aprobado por el Hospital Universitario de Santander y por el Comité de Ética de la Universidad Industrial de Santander.

\section{RESULTADOS}

\section{Generales}

Se realizaron un total de 70 procedimientos endoscópicos en 54 pacientes, discriminados en 41 endoscopias altas y 29 colonoscopias. Se les realizó endoscopia alta a 25 pacientes, solo colonoscopia a 13 y se les realizaron ambos estudios a 16 pacientes. En cuanto a variables sociodemográficas, el promedio de edad fue de 39 años, con predominio del género masculino y nivel de escolaridad en su mayoría sin sobrepasar la primaria. En la Tabla 1 se aprecia el comportamiento de variables sociodemográficas y clínicas de los pacientes. La concentración de hemoglobina tuvo un promedio de 10,9 g/dL (DE: 2,8) en hombres y de 9,6 g/dL (DE: 1,9$)$ en mujeres. En 45 pacientes $(83,3 \%)$ se encontró anemia según la definición operativa, con distribución por género de la anemia de $24(53,3 \%)$ hombres y $21(46,6 \%)$ mujeres. Se encontró un conteo promedio de linfocitos CD4 de 81,8 células por $\mathrm{mm}^{3}$. El promedio de linfocitos CD4 entre pacientes que reciben TARAE fue de 166,5 células por $\mathrm{mm}^{3}$; entre los que la habían suspendido era de $44(p=0,014)$. Comparando con quienes no habían iniciado TARAE, estos tenían un promedio de 59,7 células por $\mathrm{mm}^{3}(p=0,01)$. Por otra parte, dos terceras partes de los participantes presentaban hipoalbuminemia. La sintomatología digestiva con mayor reporte fue la diarrea y las 
lesiones orales; todos los síntomas abordados en el cuestionario son presentados en la Tabla 2 con su respectiva duración en medianas.

Tabla 1. Distribución de variables sociodemográficas, y clínicas en pacientes con $\mathrm{VIH}$

\begin{tabular}{lcccc}
\hline \multirow{2}{*}{ Variables } & \multicolumn{4}{c}{$\mathbf{n}=\mathbf{5 4}$} \\
\cline { 2 - 5 } & $\mathbf{n}$ & $\%$ & $\mathbf{9 5} \% \mathbf{I C}$ \\
\hline Género & & & & \\
$\quad$ Femenino & 23 & $42,6 \%$ & $28,9 \%$ & $56,2 \%$ \\
$\quad$ Masculino & 31 & $57,4 \%$ & $43,7 \%$ & $71,0 \%$ \\
Escolaridad & & & & \\
$\quad$ Ninguna o primaria incompleta & 10 & $18,5 \%$ & $7,8 \%$ & $29,2 \%$ \\
$\quad$ Primaria & 28 & $51,8 \%$ & $38,0 \%$ & $65,6 \%$ \\
$\quad$ Secundaria & 13 & $24,0 \%$ & $12,2 \%$ & $35,8 \%$ \\
$\quad$ Tecnología & 3 & $5,5 \%$ & $0,7 \%$ & $11,8 \%$ \\
Tiempo que lleva con el diagnóstico & & & & \\
$\quad$ Menor a 1 año & 26 & $48,1 \%$ & $34,7 \%$ & $61,9 \%$ \\
$\quad$ 1 a 5 años & 15 & $27,7 \%$ & $15,4 \%$ & $40,1 \%$ \\
$\quad$ Mayor a 5 años & 13 & $24,0 \%$ & $12,2 \%$ & $35,8 \%$ \\
Nivel de CD4 & & & & \\
$\quad \geq 200$ células por mm ${ }^{3}$ & 7 & $13,0 \%$ & $3,0 \%$ & $22,2 \%$ \\
$\quad<200$ células por mm ${ }^{3}$ & 47 & $87,0 \%$ & $77,7 \%$ & $96,2 \%$ \\
Anemia & & & & \\
$\quad$ Sí & 45 & $83,3 \%$ & $73,0 \%$ & $93,6 \%$ \\
$\quad$ No & 9 & $16,7 \%$ & $6,0 \%$ & $26,9 \%$ \\
Leucopenia & & & & \\
$\quad$ Sí & 20 & $37,0 \%$ & $23,7 \%$ & $50,3 \%$ \\
$\quad$ No & 34 & $63,0 \%$ & $49,6 \%$ & $76,2 \%$ \\
Ha recibido TARAE & & & & \\
Nunca lo ha recibido & 29 & $53,7 \%$ & 39,9 & 67,4 \\
Sí y actualmente no lo recibe & 12 & $22,2 \%$ & 10,7 & 33,6 \\
Sí y actualmente lo recibe & 13 & $24,1 \%$ & 12,2 & 35,8 \\
\hline
\end{tabular}

Tabla 2. Frecuencia de síntomas digestivos y su duración

\begin{tabular}{lccc}
\hline \multicolumn{1}{c}{ Síntoma digestivo } & $\begin{array}{c}\text { Frecuencia } \\
\text { y } \%\end{array}$ & $\begin{array}{c}\text { Días de duración } \\
\text { (mediana) }\end{array}$ & IC $95 \%$ \\
\hline Diarrea & $34(62,9 \%)$ & 7 & 7 a 20 \\
Lesiones orales & $21(38,8 \%)$ & 20 & 7 a 46 \\
Vómito & $17(31,4 \%)$ & 7 & 2 a 8 \\
Epigastralgia & $17(31,4 \%)$ & 10 & 4 a 15 \\
Dolor abdominal & $16(29,6 \%)$ & 9 & 4 a 30 \\
Odinofagia & $14(25,9 \%)$ & 7,5 & 3 a 17 \\
Nauseas & $14(25,9 \%)$ & 5 & 2 a 17 \\
Disfagia & $11(20,3 \%)$ & 10 & 4 a 30 \\
Sangrado digestivo alto & $5(9,2 \%)$ & 2 & 1 a 90 \\
Sangrado digestivo bajo & $3(5,5 \%)$ & 4 & 2 a 5 \\
\hline
\end{tabular}

\section{Hallazgos endoscópicos y patológicos de endoscopia alta}

De acuerdo con la Tabla 3, el diagnóstico con mayor reporte por el endoscopista, tanto en esófago y estómago, fue el estudio normal. Entre los hallazgos no normales más frecuentes estuvieron la esofagitis y la gastritis erosiva antral. De igual manera, el hallazgo por biopsia más frecuentemente reportado fue hallazgo de normalidad, tanto para esófago como para estómago.

\section{Hallazgos endoscópicos y patológicos de procedimiento endoscópico bajo}

De acuerdo a la Tabla 4, el diagnóstico con mayor reporte por el endoscopista, tanto en colon izquierdo como derecho, fue el estudio normal; cerca del $70 \%$ de la muestra. Sin embargo, al revisar el hallazgo por biopsia más reportado, tanto para colon izquierdo como derecho fue la colitis crónica inespecífica moderada.

\section{DISCUSIÓN}

De acuerdo con la revisión de la literatura académica, este estudio es uno de los primeros en mostrar el comportamiento de los hallazgos endoscópicos y patológicos en una serie pacientes con VIH. En Latinoamérica, el único estudio similar fue realizado en Venezuela (13), donde se estimó solamente la correlación entre la endoscopia alta y la biopsia. En cuanto a las variables sociodemográficas, la mayoría eran hombres; esta distribución por género difiere de lo reportado en el informe de ONUSIDA, que determina la prevalencia de infección por VIH tiende a distribuirse de manera similar entre hombres y mujeres (1). Por otra parte, en nuestra serie se encontró una edad promedio de 39,9 años, similar al referente nacional y regional. Así mismo, para el año 2013 el promedio de edad de los pacientes con VIH en Colombia correspondió a 39,3 años y en Santander de 39,1 años (2); dado lo anterior la enfermedad continúa siendo una enfermedad que es más prevalente en edad laboralmente activa.

La diarrea fue el síntoma gastrointestinal más frecuentemente referido, lo cual está en consonancia con lo encontrado en la literatura internacional $(14,15)$; en cuanto a sintomatología alta, en nuestra serie lo más frecuentemente reportado fueron las lesiones orales. Recientemente se publicó un trabajo en Neiva, Colombia, donde se encontró que los síntomas digestivos más frecuentes en pacientes hospitalarios con VIH eran: las lesiones orales $25,8 \%$, la diarrea $23,7 \%$ y el dolor abdominal 19,2\% (16). Un estudio en Argentina en 2004 encontró como síntomas digestivos 
Tabla 3. Comparativo de diagnósticos por endoscopia y resultado de patología en esófago y estómago

\begin{tabular}{|c|c|c|c|c|c|}
\hline \multicolumn{3}{|c|}{ Diagnóstico por endoscopia } & \multicolumn{3}{|c|}{ Diagnóstico por biopsia } \\
\hline Esófago & Frec. & $\%$ & Esófago & Frec. & $\%$ \\
\hline Estudio normal & 33 & $80,4 \%$ & Normal cambios benignos & 30 & $73,1 \%$ \\
\hline Esofagitis candidiasica Kodsi III & 4 & $9,7 \%$ & Candidiasis esofágica & 7 & $17 \%$ \\
\hline Esofagitis por citomegalovirus & 2 & $4,8 \%$ & Esofagitis crónica moderada inespecífica & 2 & $4,8 \%$ \\
\hline Esofagitis candidiasica Kodsi I & 1 & $2,4 \%$ & Candidiasis esofágica más citomegalovirus & 1 & $2,4 \%$ \\
\hline Esofagitis péptica grado C & 1 & $2,4 \%$ & Esofagitis herpética & 1 & $2,4 \%$ \\
\hline Estómago & Frec. & $\%$ & Estómago & Frec. & $\%$ \\
\hline Estudio normal & 27 & $65,8 \%$ & Normal/gastritis leve & 21 & $51,2 \%$ \\
\hline Gastritis erosiva antral & 10 & $24,3 \%$ & Gastritis crónica moderada & 11 & $26,7 \%$ \\
\hline Gastritis antral/úlcera duodenal & 1 & $2,4 \%$ & Gastritis con H. pylori & 3 & $10,1 \%$ \\
\hline Pangastritis edematosa & 1 & $2,4 \%$ & Gastritis crónica severa & 1 & $2,4 \%$ \\
\hline Úlcera antral & 1 & $2,4 \%$ & Gastritis crónica atrófica leve & 1 & $2,4 \%$ \\
\hline \multirow[t]{3}{*}{ Úlcera duodenal } & 1 & $2,4 \%$ & Gastritis crónica severa con metaplasia & 1 & $2,4 \%$ \\
\hline & & & Gastritis por histoplasma & 1 & $2,4 \%$ \\
\hline & & & Úlcera por citomegalovirus & 1 & $2,4 \%$ \\
\hline
\end{tabular}

Tabla 4. Comparativo de diagnósticos por endoscopia y resultado de patología en colon

\begin{tabular}{|c|c|c|c|c|c|}
\hline \multicolumn{3}{|c|}{ Diagnóstico por endoscopia } & \multicolumn{3}{|c|}{ Diagnóstico por biopsia } \\
\hline Colon izquierdo & Frec. & $\%$ & Colon izquierdo & Frec & $\%$ \\
\hline Estudio normal & 20 & $68,9 \%$ & Colitis crónica inespecífica moderada & 13 & $44,8 \%$ \\
\hline Colitis por citomegalovirus & 4 & $13,7 \%$ & Normal/colitis leve & 6 & $20,6 \%$ \\
\hline Colitis inespecífica & 2 & $6,9 \%$ & Colitis por citomegalovirus & 5 & $17,2 \%$ \\
\hline Colitis por parasitismo intestinal & 1 & $3,4 \%$ & Colitis crónica inespecífica severa & 2 & $6,9 \%$ \\
\hline Enfermedad diverticular & 1 & $3,4 \%$ & Colitis por histoplasma & 1 & $3,4 \%$ \\
\hline \multirow[t]{2}{*}{ Hemorroides internas } & 1 & $3,4 \%$ & Colitis ulcerativa & 1 & $3,4 \%$ \\
\hline & & & Úlcera por histoplasma y citomegalovirus & 1 & $3,4 \%$ \\
\hline Colon derecho & Frec. & $\%$ & Colon derecho & Frec. & $\%$ \\
\hline Estudio normal & 21 & $72,4 \%$ & Colitis crónica inespecífica moderada & 15 & $51,7 \%$ \\
\hline Colitis por citomegalovirus & 4 & $13,7 \%$ & Colitis por citomegalovirus & 5 & $17,2 \%$ \\
\hline Colitis inespecífica & 2 & $6,9 \%$ & Normal/colitis leve & 5 & $17,2 \%$ \\
\hline Lesión angiomatosa & 1 & $3,4 \%$ & Colitis crónica inespecífica severa & 2 & $6,9 \%$ \\
\hline \multirow[t]{2}{*}{ Úlcera por M. tuberculosis } & 1 & $3,4 \%$ & Colitis ulcerativa & 1 & $3,4 \%$ \\
\hline & & & Úlcera por histoplasma & 1 & $3,4 \%$ \\
\hline
\end{tabular}

altos más frecuentes: la sensación de acidez 73,5\%, el dolor epigástrico 73,5\% y la distensión postprandial 65,7\% (17). En Bélgica, se encontró que las indicaciones para realizar endoscopia digestiva alta fueron: incomodidad abdominal $31,4 \%$, otros síntomas en $28,3 \%$ y síntomas de reflujo $16,1 \%$ (18). Por lo referido anteriormente, los signos y síntomas digestivos en pacientes con VIH/SIDA toman una tendencia muy variada; por lo tanto deben ser estudiados adecuadamente para poder definir un diagnóstico específico.
No existen muchas publicaciones que desglosen la sensibilidad de la endoscopia y colonoscopia como ayuda diagnóstica y mucho menos en pacientes con VIH. Por lo general la tendencia es presentar artículos donde el rendimiento diagnóstico se enfoca para patógenos en específico, por esta razón no es recomendable en este estudio abordar el análisis de los datos en términos de sensibilidad especificidad y medidas afines, dado por la heterogeneidad de diagnósticos encontrados. Por eso, nos enfocamos únicamente en la des- 
cripción de los hallazgos endoscópicos y patológicos, y en alguna medida observar el porcentaje de comportamiento del diagnóstico de normalidad reportado por el endoscopista y por anatomía patológica en cada uno de los órganos abdominales.

Nuestros hallazgos reportados por anatomía patológica en esófago fueron en su mayoría estudio normal y en segunda instancia candidiasis. Llama la atención que no se diagnosticó esofagitis eosinofílica, entidad de la cual se conoce cada vez más y que puede llegar a tener una incidencia en la población general de un $0,4 \%$ y la prevalencia puede llegar a ser del $54 \%$ en pacientes con antecedente de impactación por alimentos (20). En otras series se han encontrado como diagnósticos patológicos frecuentes en esófago: cándida, citomegalovirus, virus del herpes simple y virus del papiloma humano $(3,13,19,20)$. En nuestro estudio, con respecto a la capacidad de la endoscopia de detectar normalidad, al confrontar con la biopsia, en la Tabla 3 vimos que la normalidad fue similar entre la visión por endocopista y patólogo. Lo anterior se podría explicar por la cantidad limitada de oportunismos a este nivel; sin embargo, es necesario incluir la biopsia dentro del estudio del paciente dado algunas patologías como la esofagitis eosinofílica puede cursar con mucosa normal a la vista del endoscopista (21).

En cuanto a estómago, los referentes bibliográficos señalan como oportunismos gástricos al Cytomegalovirus y Mycobacterium avium, y como no oportunistas al Helicobacter pylori (H. pylori) (22). Además se suelen encontrar lesiones neoplásicas como el linfoma no Hodgkin y el sarcoma de Kaposi $(6,18)$. En el presente estudio, los hallazgos endoscópicos en estómago más frecuentes fueron los relacionados a mucosa normal, y los diagnósticos patológicos encontraron oportunismos como el histoplasma y citomegalovirus. No se encontró infección por micobacterias ni neoplasias gástricas; es importante mencionar que se encontró $H$. pylori en el $8 \%$ de la muestra, también cabe anotar que los tres casos de $H$. pilory se habían leído como normales en el estudio endoscópico. La prevalencia de H. pylori en la población con VIH estudiada fue menor a la reportada en la población sin $\mathrm{VIH}$ en Colombia (23). Se destaca que no hay referentes en la literatura sobre prevalencia en Colombia de $H$. pylori en población con VIH. Debido a las limitaciones del presente estudio, se requieren estudios con inclusión de mayor población y comparación con grupos control para determinar adecuadamente la prevalencia de $H$. pylori en la población con $\mathrm{VIH}$ en nuestro medio.

De acuerdo a la Tabla 4, en el colon derecho e izquierdo, a diferencia del esófago y el estómago, el porcentaje de reporte de normalidad según el estudio anatomopatológico es mucho menor que el ofrecido por el diagnóstico del endoscopista; esto pudiera explicarse porque el colon normalmente tiene mayor cantidad de bacterias, mayor cantidad de tejido linfoide, lo cual pudiera explicar patologías no oportunistas como causales de la sintomatología. Aunque sí es claro que la cantidad de patologías y oportunismos es mayor en colon que en esófago y estómago (19). A su vez, es importante enfatizar en realizar la biopsia diagnóstica, porque la mucosa en apariencia sana a la vista del endoscopista en pacientes con sintomatología digestiva puede corresponder no solo a agentes oportunistas sino a patologías diferentes como la colitis colagenosa (24-26). Otra posible explicación para la alta frecuencia de hallazgos patológicos inespecíficos en colon Colitis inespecífica es que entidades como la colitis colagenosa, la colitis linfocitaria y la gastroenteritis eosinofílica son diagnosticadas poco frecuentemente en nuestro medio y los patólogos están poco acostumbrados a su reporte (27).

Entre las limitaciones del presente estudio, no se realizó microscopia electrónica, inmunohistoquímica, ni cultivos, ni biología molecular; dado lo anterior algunos informes de normalidad pudieran realmente tener alguna alteración patológica. Como ejemplo, la microscopia electrónica puede ser usada para identificar infecciones protozoarias del intestino delgado por Cryptosporidium y Microsporidia; por otra parte la inmunohistoquimica, FISH y PCR ayudarían a identificar infecciones virales por citomegalovirus o por el virus del herpes simple $(6,28)$. Otra limitación fue que en este estudio no se realizó la carga viral en los pacientes. Es importante anotar que la viremia puede explicar acción directa del virus en las mucosas como causal de la sintomatología si se descartan otras causas. Finalmente, es posible que el tamaño de muestra no sea el ideal para realizar generalizaciones de hallazgos, teniendo en cuenta la heterogeneidad en los diagnósticos.

\section{CONCLUSIONES}

Los hallazgos endoscópicos y patológicos concuerdan con la frecuencia de presentación en el tracto gastrointestinal reportada en la literatura, aunque no se encontraron neoplasias, ni algunos agentes infecciosos como: micobacterias, adenovirus, coronavirus, entre otros. El oportunista más frecuente en el esófago fue la cándida y en el colon, el citomegalovirus; este último fue globalmente el oportunista más frecuentemente encontrado. Solamente se presentó una buena correlación para diagnosticar normalidad entre la endoscopia y la patología en esófago; a diferencia de estómago y colon, donde la disparidad es evidente. Por lo anterior, de acuerdo al estado del arte, se recomienda que, independientemente de la observación del endoscopista, se requiere que en los pacientes con VIH/SIDA y síntomas digestivos que sean llevados a estudios endoscópicos, se tomen biopsias de manera protocolizada. Además, dicho 
estudio patológico debe contar con protocolos de lectura completos, que tengan en cuenta las etiologías oportunistas y no oportunistas mediante tinciones convencionales, microscopia electrónica e inmunohistoquímica. Con lo cual se podría llegar a un diagnóstico etiológico preciso y orientar la terapéutica de forma adecuada en los pacientes, lo que evitaría hospitalizaciones y desenlaces clínicos severos o fatales.

\section{Conflicto de intereses}

Los autores declaran no tener conflicto de intereses.

\section{Financiación}

No se contó con financiación especial para la ejecución de este trabajo.

\section{REFERENCIAS}

1. ONUSIDA, Informe sobre la Epidemia Mundial de SIDA 2012. [Internet]. [citado 24 de mayo de 2016]. Recuperado a partir de: http://www.unaids.org/sites/ default/files/media_asset/20121120_UNAIDS_Global_ Report_2012_with_annexes_es_1.pdf

2. CAC.CO_20150814_LIBRŌVĪH2014_V-0.0.VERSIÓN FINAL---.pdf [Internet]. [citado 24 de mayo de 2016]. Recuperado a partir de: https://cuentadealtocosto. org/site/images/Publicaciones/CAC.CO_20150814 LIBROVIH2014_V-0.0.VERSI\%C3\%93N\%20FINAL---.pdf

3. Torre D, Speranza F, Martegani R. Impact of highly active antiretroviral therapy on organ-specific manifestations of HIV-1 infection. HIV Med. 2005;6(2):66-78.

4. Knox TA, Spiegelman D, Skinner SC, Gorbach S. Diarrhea and abnormalities of gastrointestinal function in a cohort of men and women with HIV infection. Am J Gastroenterol. 2000;95(12):3482-9.

5. Fuyuno Y, Yamazaki K, Takahashi A, Esaki M, Kawaguchi T, Takazoe M, et al. Genetic characteristics of inflammatory bowel disease in a Japanese population. J Gastroenterol. 2015.

6. Bhaijee F, Subramony C, Tang S-J, Pepper DJ. Human immunodeficiency virus-associated gastrointestinal disease: common endoscopic biopsy diagnoses. Patholog Res Int. 2011;2011:247923.

7. Zeitz M, Ullrich R, Schneider T, Kewenig S, Riecken E. Mucosal immunodeficiency in HIV/SIV infection. Pathobiology. 1998;66(3-4):151-7.

8. Kearney DJ, Steuerwald M, Koch J, Cello JP. A prospective study of endoscopy in HIV-associated diarrhea. Am J Gastroenterol. marzo de 1999;94(3):596-602.

9. Sayilir A, Kurt M, Kekilli M, Onal IK, Beyazit Y, Turhan N, et al. Diagnostic value of terminal ileum biopsy in chronic diarrhea with normal endoscopic appearance. J Dig Dis. junio de $2011 ; 12(3): 188-92$.
10. Nishimura S, Nagata N, Shimbo T, Asayama N, Akiyama J, Ohmagari N, et al. Factors associated with esophageal candidiasis and its endoscopic severity in the era of antiretroviral therapy. PLoS ONE. 2013;8(3):e58217.

11. Lundell LR, Dent J, Bennett JR, Blum AL, Armstrong D, Galmiche JP, et al. Endoscopic assessment of oesophagitis: clinical and functional correlates and further validation of the Los Angeles classification. Gut. 1999;45(2):172-80.

12. Satsangi J, Silverberg MS, Vermeire S, Colombel J-F. The Montreal classification of inflammatory bowel disease: controversies, consensus, and implications. Gut. junio de 2006;55(6):749-53.

13. Fernández S, Lecuna V, Ruiz ME. Comparación en el tracto digestivo superior entre los hallazgos endoscópicos y la biopsia en pacientes con síndrome de inmunodeficiencia adquirida (sida) con más de 200 células cd4 y menos de 200 células cd4. Gen. 2007;61(1):14-20.

14. Feasey NA, Healey P, Gordon MA. Review article: the aetiology, investigation and management of diarrhoea in the HIV-positive patient. Aliment Pharmacol Ther. 2011;34(6):587-603.

15. Ríos N, Núñez A, Chamorro C. Frecuencia de alteraciones gastrointestinales en pacientes con SIDA internados en el IMT en el año 2009 y 2010. Revista del Instituto de Medicina Tropical. 2014;6(2):6-10.

16. Agudelo González S, Murcia Sánchez F, Salinas D. Osorio J. Infecciones oportunistas en pacientes con VIH en el hospital universitario de Neiva, Colombia. 2007-2012. Infectio; 2015;52-59.

17. Olmos MA, et al. Evaluación endoscópica de pacientes VIH positivos con síntomas digestivos altos. Acta Gastroenterológica Latinoamericana. 2004;34:120-6.

18. Nkuize M, De Wit S, Muls V, Arvanitakis M, Buset M. Upper gastrointestinal endoscopic findings in the era of highly active antiretroviral therapy. HIV Med. 2010;11(6):412-7.

19. Al Anazi AR. Gastrointestinal opportunistic infections in human immunodeficiency virus disease. Saudi J Gastroenterol. abril de 2009; 15(2):95-9.

20. Furuta GT, Katzka DA. Eosinophilic Esophagitis. N Engl J Med. 2015;373(17):1640-8.

21. Beltrán M, Constanza; García M, Raimundo; Espino E, alberto y Silva A, Claudia. Esofagitis Eosinofílica: Una entidad emergente. Rev. Otorrinolaringol. Cir. Cabeza Cuello. 2009;69(3):287-98.

22. Nevin DT, Morgan CJ, Graham DY, Genta RM. Helicobacter pylori gastritis in HIV-infected patients: a review. Helicobacter. octubre de 2014;19(5):323-9.

23. Gómez, Martín et al. Erradicación del Helycobacter pylori: encuesta realizada por la Asociación Colombiana de Gastroenterología. Rev Col Gastroenterol. 2015;30(1):25-31.

24. Narabayashi K, Murano M, Egashira Y, Noda S, Kawakami $\mathrm{K}$, Ishida K, et al. Endoscopic and histopathological evaluation of collagenous colitis. Digestion. 2012;85(2):136-40.

25. Fernandes ER, Pagliari C, Tuon FF, de Andrade Junior HF, Averbach M, Duarte MIS. Chronic colitis associated with $\mathrm{HIV}$ infection can be related to intraepithelial infiltration of 
the colon by CD $8+\mathrm{T}$ lymphocytes. Int J STD AIDS. agosto de 2008; 19(8):524-8.

26. Arevalo F, Arias SC, Monge E. Biopsia de colon: características histológicas en diferentes tipos de Colitis Crónica. Rev gastroenterol Perú. 2008;28(2):140-9.
27. Ingle SB, Hinge Ingle CR. Eosinophilic gastroenteritis: an unusual type of gastroenteritis. World J Gastroenterol. 21 de agosto de 2013;19(31):5061-6.

28. Huppmann AR, Orenstein JM. Opportunistic disorders of the gastrointestinal tract in the age of highly active antiretroviral therapy. Hum Pathol. diciembre de 2010;41(12):1777-87. 